\title{
Real-time protein unfolding: a method for determining the kinetics of native protein denaturation using a quantitative real-time thermocycler
}

\author{
Kyle K. Biggar*, Neal J. Dawson*, and Kenneth B. Storey \\ Institute of Biochemistry \& Department of Biology, Carleton University, Ottawa, Ontario, Canada \\ ${ }^{*}$ K.K.B. and N.J.D. contributed equally to this article.
}

BioTechniques 53:231-238 (October 2012) doi 10.2144/0000113922

Keywords: protein stability; urea; qRT-PCR; SYPRO Orange; fluorescence; lysozyme; hexokinase

Supplementary material for this article is available at www.BioTechniques.com/article/113922

Protein stability can be monitored by many different techniques. However, these protocols are often lengthy, consume large amounts of protein, and require expensive and specialized instruments. Here we present a new protocol to analyze protein unfolding kinetics using a quantified real-time thermocycler. This technique enables the analysis of a wide range of denaturants (and their interactions with temperature change) on protein stability in a multi-well platform, where samples can be run in parallel under virtually identical conditions and with highly sensitive detection. Using this set-up, researchers can evaluate the half-maximal rate of protein denaturation $\left(\mathrm{K}_{\mathrm{nd}}\right)$, maximum rate of denaturation $\left(\mathrm{D}_{\max }\right)$, and the cooperativity of individual denaturants in protein unfolding ( $\mu$-coefficient). Both lysozyme and hexokinase are used as model proteins and urea as a model denaturant to illustrate this new method and the kinetics of protein unfolding that it provides. Overall, this method allows the researcher to explore a large number of denaturants, at either constant or variable temperatures, within the same assay, providing estimates of denaturation kinetics that have been previously inaccessible.

The limits of survival for many living organisms are marked by extreme environmental conditions: for example, 0 to 12.5 for $\mathrm{pH}$ and approximately $-18^{\circ} \mathrm{C}$ to $121^{\circ} \mathrm{C}$ for temperature (1). While some organisms that live in extreme environments can evade the stress or employ defensive mechanisms that allow them to sustain favorable intracellular conditions, other organisms must adapt their intracellular molecules for survival. For example, the intracellular $\mathrm{pH}$ of many acidophiles, neutrophiles and alkaliphiles are regularly maintained at around 6.5, 7.5, and 9.5, respectively (2). To maintain proper functioning of their intracellular proteins, these organisms display structural adaptations that influence both protein stability and activity (3). Apart from the ability of some species to exist at high and low intracellular $\mathrm{pH}$, many organisms have the ability to tolerate extreme temperatures. For example, many organisms have successfully colonized cold environ- ments and have evolved mechanisms to overcome the life-endangering consequences of low temperature with structural and functional adaptations of their macromolecules that include adaptations of protein structure, stability and posttranslational modifications (4). Likely candidates for adaptive changes are those proteins with crucial functions necessary for survival (5-7). Consequently, there is great interest in understanding the mechanisms involved in stabilizing protein structure, not only for extremophiles, but for modified protein structures in general.

It has been known for many years that proteins can be unfolded in aqueous solution by high concentrations of denaturants, such as guanidine hydrochloride $(\mathrm{GnHCl})$ or urea. Denaturation using these chemicals are one of the primary methods of determining protein structure, comparing the stability of modified to native proteins. Denaturants alter the equilibrium between the native (folded) and denatured (unfolded) states of the protein:

$$
\mathrm{N} \leftrightarrow \mathrm{D}
$$

where $N$ is the concentration of protein in the folded or native conformation, and $D$ is the concentration of protein in the unfolded or denatured state at a particular denaturant concentration. The relative concentrations of $N$ and $D$ are typically determined with the use of either (i) fluorescent probes to monitor the conformational state of the protein or (ii) proteolysis combined with SDS-PAGE (known as pulse proteolysis) to monitor relative amounts of denatured (unfolded) or non-denatured (folded) protein. Although this type of experiment can provide information about the free energy of protein stability, it does not provide information regarding the rate of protein denaturation. Determining the kinetics of native protein denaturation could provide the researcher with detailed information regarding protein 


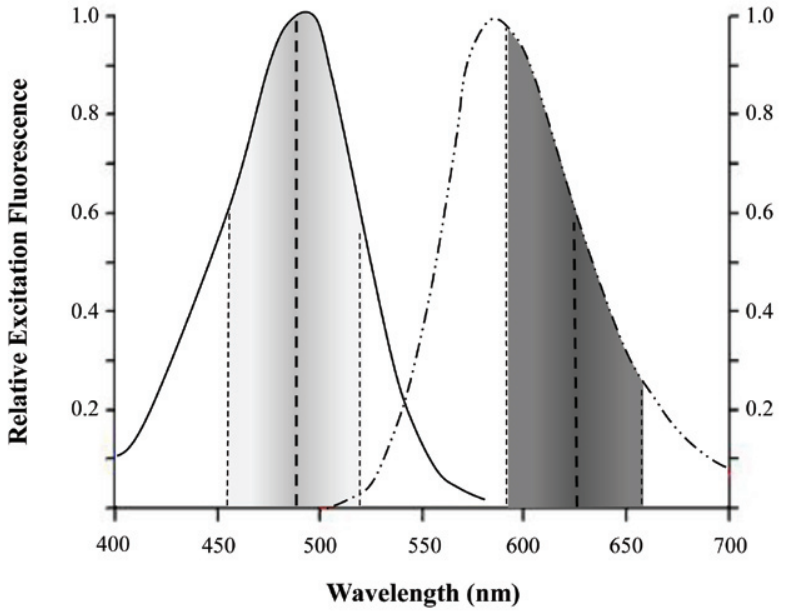

Figure 1. Excitation (solid) and emission (dashed) spectra of SYPRO orange fluorescent dye in BSA. Shaded areas represent the relative range of light transmitted through the BioRad $485 \pm 30 \mathrm{~nm}$ excitation and $625 \pm 30 \mathrm{~nm}$ emission filters used in this experiment. This figure is modified from Invitrogen Life Technologies product information (Cat. No. S-6650).

\section{High Efficiency Cell Disrupter Mini BeadBeater-24}

- Homogenizes 24 samples in $2 \mathrm{ml}$. vials.

- Proven more efficient horizontal vial shaking.

- High energy, variable speed motor. No motor cool-down time.

- Spores, bacterial yeast and tissue.

- Totally sealed system. No clean-up. 3. 5 BIOSPEC PRODUCTS

P.O. Box 788 - Bartlesville, OK 74005

Phone: 1-800-617-3363 • http://www.biospec.com structure and the influence of various effectors on protein stability and unfolding rate.

The determination of protein stability is an essential step in characterizing protein structure. There are many traditional techniques, such as fluorescence, circular dichroism spectroscopy, hydrogen exchange-mass spectroscopy, differential scanning fluorometry (DSF), protein crystallization, and pulse proteolysis used to determine the structural stability of proteins and enzymes. These methods determine stability by monitoring conformational changes induced by either thermal or chemical denaturation. However, with the exception of DSF, these methods require substantial amounts of pure protein and expensive specialized instrumentation. Unfortunately, although DSF is able to utilize low amounts of protein, the technique only monitors the thermal stability of protein structure as a sample is exposed to increasing temperature (8). In the present study, we expanded the method of DSF to evaluate denaturation kinetics of proteins when exposed to a denaturant at either constant or varying temperatures.

Existing DSF methods work by utilizing a modified qRT-PCR thermocycler to monitor thermal unfolding of a protein in the presence of a hydrophobic fluorescent dye (typically SYPRO Orange). As a result, the use of DSF is applicable to a wide range of proteins that contain hydrophobic regions within the protein structure. The presence of a hydrophobic region is critical, since the SYPRO Orange dye used for DSF is highly fluorescent upon binding to hydrophobic sites on unfolded proteins. The typical analysis conducted using DSF is to determine the change in fluorescence intensity as a function of increasing temperature; this typically generates a sigmoidal curve that can be described by a two-state transition (8). The relatively high wavelength for excitation of SYPRO Orange $(488 \mathrm{~nm})$ also decreases the likelihood that small molecules would interfere with the optical properties of the dye. In the present study, we present important modifications to the DSF procedure to obtain real-time kinetic analysis of protein unfolding. Our modifications expand the DSF technique by altering the experimental protocol, allowing the researcher to obtain real-time fluorescent data over a constant (or gradient) temperature and collect data at regular intervals (between $1 \mathrm{~s}$ and 99 min 59 s). By combining the use of (i) a 96-well PCR microplate, (ii) relatively small sample volumes $(20 \mu \mathrm{L})$, (iii) a short procedure time, and (iv) the ability to monitor denaturation at various temperatures (typically ranging from $4.0^{\circ} \mathrm{C}$ $-99.0^{\circ} \mathrm{C}$ on a gradient-enabled qRT-PCR thermocycler), this protocol allows researchers to combine multiple experimental conditions into a single experiment, reducing both time and technical variation. This method is a highly accurate and reproducible way to assess protein stability and determine the rate of protein denaturation under environmental conditions including variable $\mathrm{pH}$, denaturants (e.g urea and $\mathrm{GnHCl}$ ) and stabilizers (e.g glycerol and trehalose).

\section{Materials and methods}

\section{Equipment preparation}

As a result of a broad range of excitation and emission fluorescence, SYPRO Orange (Invitrogen, Burlington, ON, Canada, Cat. No. S-6650) can be monitored in quantitative RT-PCR instruments using the filters commonly provided with the machines: FAM $(485 \mathrm{~nm})$ and ROX $(625 \mathrm{~nm})$ for excitation and emission, respectively (Figure 1). For the presented set of experiments, we modified a BioRad MyIQ2 thermocycler (BioRad, Hercules, CA, USA, Cat. No. 
A

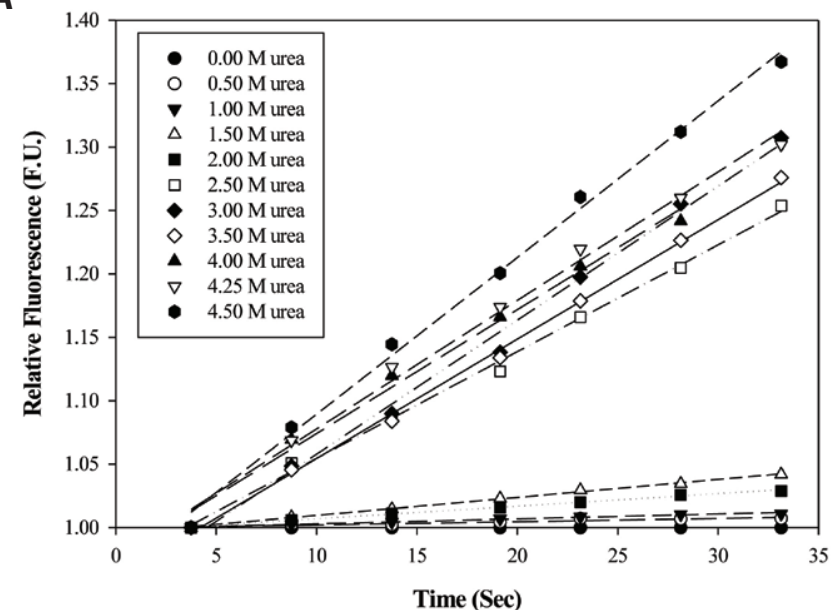

B

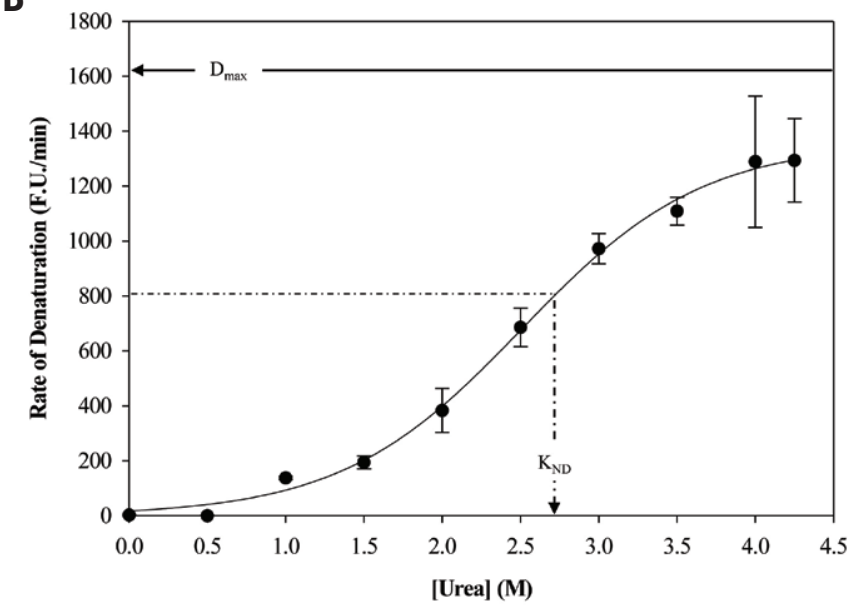

C

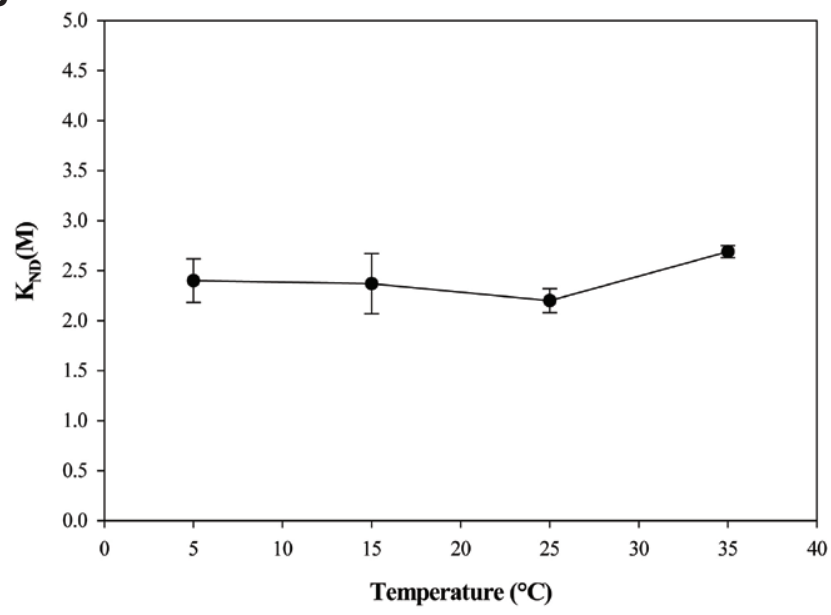

Figure 2. The effect of urea on the rate of hen white lysozyme (HEWL) unfolding. (A) Representative plot $(n=1)$ of HEWL denaturation assessed via changes in fluorescence units (F.U.) relative to $0 \mathrm{M}$ urea. Fluorescence is measured as the result of SYPRO orange binding over the initial seconds of exposure to urea. (B) Effect of urea on the velocity of HEWL denaturation as determined by the slope of relative fluorescence over time at various concentrations of urea in potassium phosphate buffer. (C) Effect of urea on the $\mathrm{K}_{\mathrm{nd}}$ of $\mathrm{HK}$ protein denaturation in samples incubated at various temperatures. $\mathrm{HEWL}$ samples were incubated at $25^{\circ} \mathrm{C}$ in various concentrations of urea in potassium phosphate buffer. Fluorescence (excitation $\lambda=485 \mathrm{~nm}$; emission $\lambda=625 \mathrm{~nm}$ ) was measured in a modified BioRad MyIQ2 thermocycler. Data for plots $(B)$ and $(C)$ are means $\pm \operatorname{SEM}(n=4$ trials). For temperature studies, "a" indicates significantly different from the $5^{\circ} \mathrm{C}$ value, "b" indicates significantly different from $15^{\circ} \mathrm{C}$, and " $\mathrm{C}$ " indicates significantly different from $25^{\circ} \mathrm{C}(P<0.05)$.
170-9790), installing the appropriate filters into a separate channel than the one utilized for SYBR-green based experiments. This allowed continued functionality for both qRT-PCR and protein denaturation experiments. A pure dye calibration was carried out for SYPRO Orange. No further modifications were carried out to the instrument.

Protein denaturation kinetics Lysozyme purified from hen egg whites (HEWL) (Worthington Biochemical Corp., Lakewood, NJ, USA, Cat. No. LS002931) was diluted to a concentration of $25 \mathrm{mg} / \mathrm{mL}$ in potassium phosphate buffer $(100 \mathrm{mM}$ potassium phosphate, $\mathrm{pH} 7.0,150 \mathrm{mM} \mathrm{NaCl}$ ), and hexokinase (HK) purified from Saccharomyces cerevisiae (Sigma Aldrich, St. Louis, MO, USA, Cat. No. H-5500) was diluted to a concentration of $0.2 \mathrm{mg} / \mathrm{mL}$ in potassium phosphate buffer. Optimal protein concentrations were determined using the method outlined below and were found to provide a suitable doseresponse relationship when compared with other protein concentrations. This buffer was previously shown to be suitable for studies of protein denaturing conditions and is commonly used in experiments involving SYPRO Orange (8). The SYPRO Orange dye was diluted to a $40 \times$ stock (based on the $5000 \times$ stock solution as supplied by Invitrogen) in potassium phosphate buffer and used from this concentration for all experiments.

All experiments using the qRT-PCR thermocycler were carried out in 96-well thin-walled unskirted PCR microplates (BioRad, Cat. No. MLP-9601). All incubations with urea were conducted at 11 different final concentrations ranging from 0 to $4.5 \mathrm{M}$ in potassium phosphate buffer. Each sample consisted of $12.5 \mu \mathrm{L}$ of potassium phosphate buffer containing various concentration of urea, $5 \mu \mathrm{L}$ of purified protein and $2.5 \mu \mathrm{L}$ of $40 \times$ SYPRO Orange dye for a final volume of $20 \mu \mathrm{L}$. Fluorescent measurements were initiated $\sim 5 \mathrm{~s}$ after the addition of protein to the samples. Fluorescent reads were taken every $5 \mathrm{~s}$ at $5^{\circ} \mathrm{C}, 15^{\circ} \mathrm{C}$, $25^{\circ} \mathrm{C}$, and $35^{\circ} \mathrm{C}$ for $50 \mathrm{~min}$, using the manufacturer-supplied software (version 3.0.6070). Temperature incubations were carried out in separate experiments. Data were analyzed using SigmaPlot v.11 software (Systat Software, Chicago, IL, USA). The change in fluorescence was plotted using a simple scatter plot. The whole unfolding curve was measured in the experiment and fluorescence was found to rapidly increase initially and 
then reach a plateau (Supplementary Figure S1). The initial unfolding rates of the curve were truncated and analyzed using a linear regression of fluorescence at various concentrations of urea. All experiments were carried out at a concentration of SYPRO Orange within a linear region of fluorescence that displayed no significant changes in protein unfolding rate. The concentration of SYPRO used in the study is comparable to other protein unfolding studies (8).

The resulting slopes were plotted on a second graph using the Kinetics $v$ 3.5.1 program and analyzed using the Hill Equation (9). From this, $K_{n d}, D_{\text {max }}$, and $\mu$-coefficient values were calculated. Respectively, these values were defined as the concentration of denaturant (urea in this case) that resulted in a half-maximal rate of native protein denaturation $\left(\mathrm{K}_{\mathrm{nd}}\right)$, the calculated maximum rate of protein denaturation $\left(\mathrm{D}_{\max }\right)$, and the extent of denaturant cooperativity ( $\mu$-coefficient) calculated in a similar manner to that of a Hill coefficient. Data are expressed as mean \pm SEM from multiple trials. Statistical testing used Student's $t$-test.

\section{Results and discussion}

Studies characterizing protein unfolding can help researchers to understand general questions of protein stability under various conditions. The methodology described in this study extends the range of options for researchers interested in investigating protein stability. By using modified qRT-PCR equipment, it is possible to analyze the effects of a wide range of denaturants (and their interactions with temperature change) on protein stability in a microplate platform where multiple samples can be run in parallel under virtually identical conditions and with highly sensitive detection. Using this set-up, researchers can evaluate the half-maximal rate of protein denaturation $\left(\mathrm{K}_{\mathrm{nd}}\right)$, maximum rate of protein denaturation $\left(\mathrm{D}_{\text {max }}\right)$, and the cooperativity of individual denaturants in protein unfolding ( $\mu$-coefficient). As a protein unfolds, it exposes hydrophobic residues that are able to interact with SYPRO Orange dye, resulting in an increase in fluorescent emission that can be detected by qRT-PCR optics. In the present study we used two model proteins (lysozyme and hexokinase) and a model denaturant (urea) to demonstrate this new method and the kinetics of protein unfolding that it can supply. HEWL was chosen as a model protein as it is a wellstudied monomer that has been shown
A

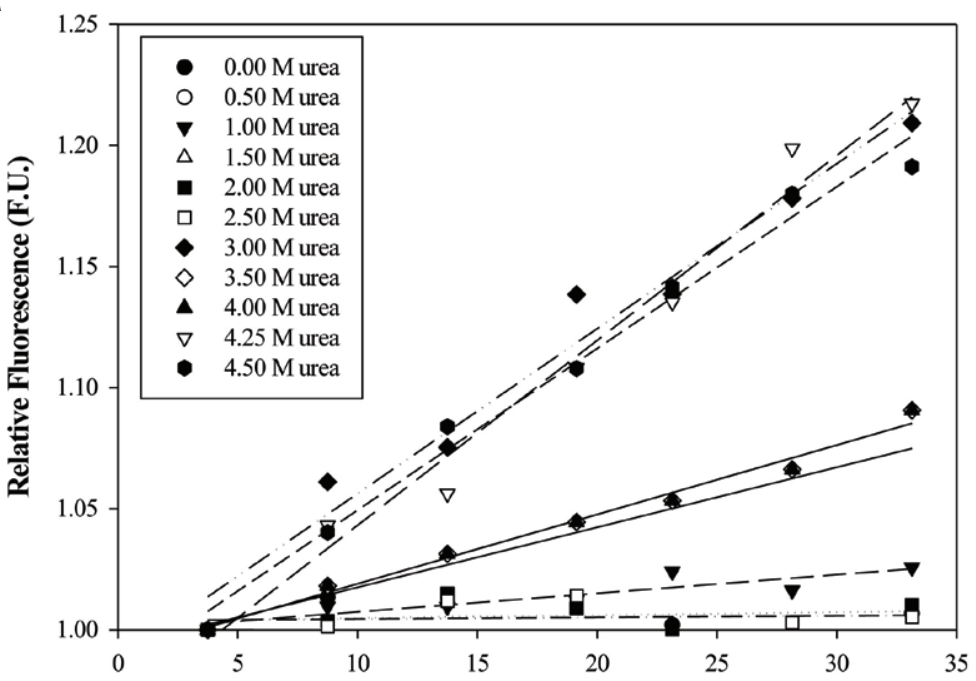

B

Time (Sec)

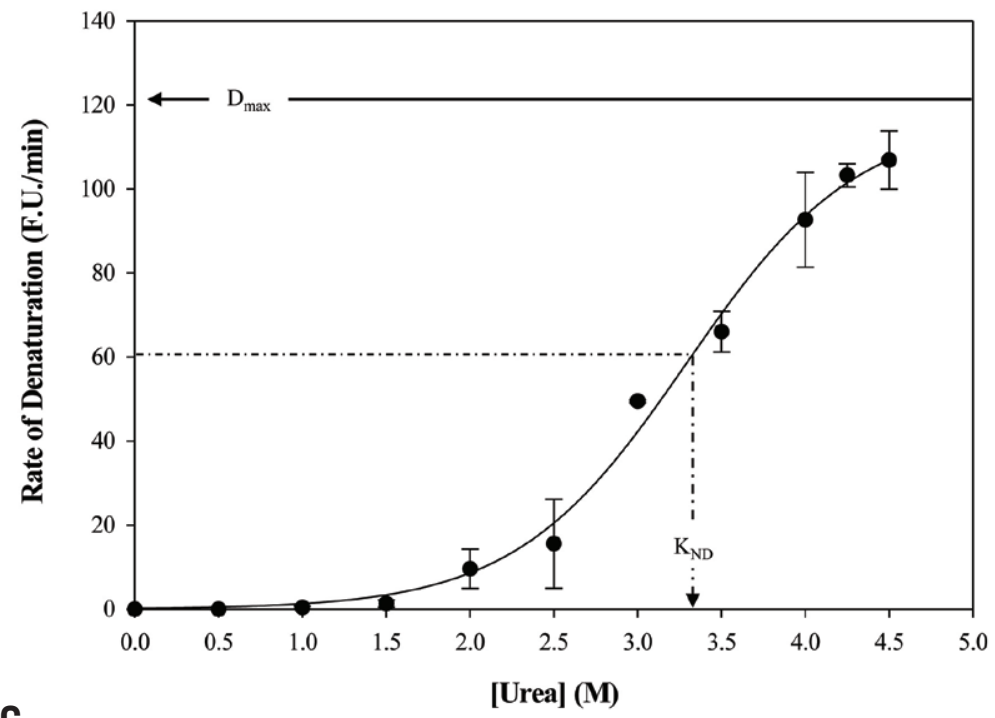

C

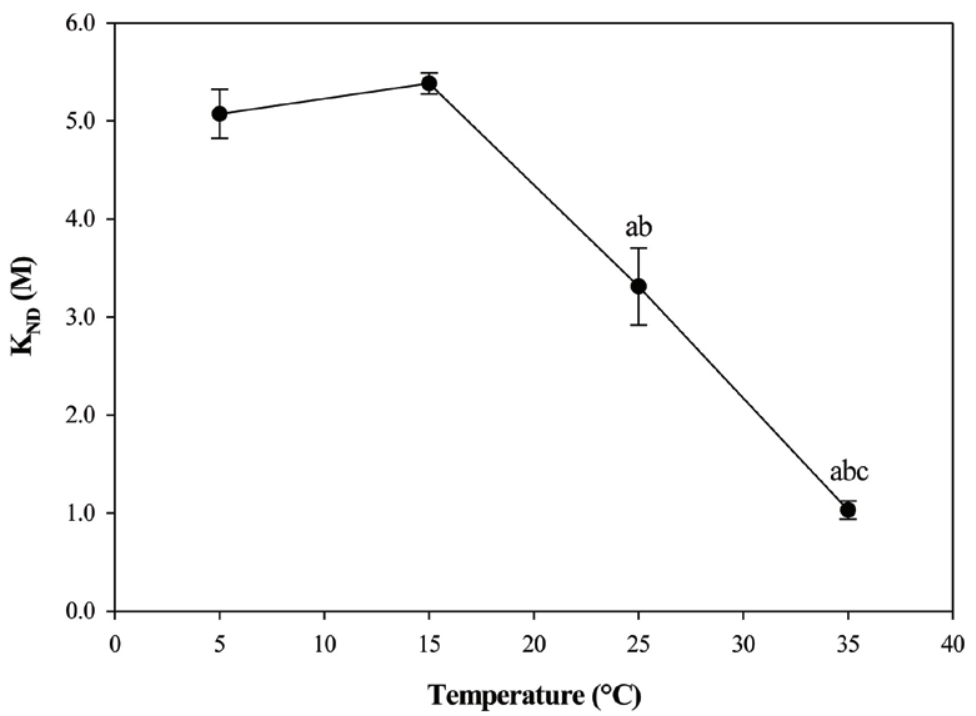

Figure 3. The effect of urea on the rate of hexokinase unfolding. Other information as in Figure 2. 
to unfold in a relatively simplistic model of a two-state transition, while yeast hexokinase $(\mathrm{HK})$ is a dimer also been shown to undergo a two-state transition of unfolding $(10-11)$.

To determine the effects of urea concentration on protein unfolding rates, protein samples were incubated at various urea levels in the presence of SYPRO Orange dye. At each urea concentration, the relative rate of unfolding of the protein was recorded as an increase in the fluorescence of SYPRO Orange over time. The changes in fluorescent values were then plotted as a function of incubation time. Data points were truncated to display only the initial linear portion of the denaturation curve (Figure 2A and 3A). The slope of this relationship was then calculated for each urea concentration using a linear regression of fluorescence with respect to incubation time. Slopes increased with increasing urea concentration. Importantly, it should be noted that this technique will not be able to measure the initial rates of rapidly unfolding proteins as there is a dead time of $\sim 5 \mathrm{~s}$ between the addition of protein and the initial fluorescent reads from the thermocycler. To determine the rate of HEWL and HK denaturation in urea, each resulting slope (calculated above) was then plotted against the concentration of urea. These values gave a sigmoidal plot that was analyzed using the Hill equation (Figure 2B and 3B) (12). Interestingly, we have found that our plots of unfolding rates for HEWL are similar to those by Laurents and Baldwin, whose study used tryptophan fluorescence; however, comparable rates of unfolding could not be obtained (13).

The concentration of urea that results in half-maximal rate of protein denaturation is defined as the kinetic constant of native denaturation $\left(\mathrm{K}_{\text {nd }}\right)$. The $\mathrm{K}_{\text {nd }}$ at $25^{\circ} \mathrm{C}$ was determined to be $2.69 \pm$ $0.06 \mathrm{M}(n=4)$ for HEWL and $3.31 \pm$ $0.22 \mathrm{M}(n=4)$ for HK. As urea concentration increases, protein will denature at a faster rate until a maximum rate is reached. This maximum rate, $\mathrm{D}_{\max }$, can be calculated from an asymptote determined from the Hill equation. This $\mathrm{D}_{\text {max }}$ value, therefore, represents the upper limit of the rate of protein denaturation (the change in fluorescent units per minute or $\Delta$ F.U./min). From the present data, a $\mathrm{D}_{\max }$ of $1636 \pm 147 \Delta \mathrm{F} . \mathrm{U} . / \mathrm{min}$ was calculated for HEWL and $124 \pm 8 \Delta$ F.U./min was calculated for $\mathrm{HK}$ denaturation by urea at $25^{\circ} \mathrm{C}$.

The extent of cooperativity of the denaturant can also be uncovered when calculating the apparent Hill coefficient. As urea molecules interact with a protein there is a disruption of intra-molecular interactions. Upon the disruption of these intra-molecular forces, the protein becomes increasingly accessible to subsequent urea molecules. This results in a cooperative effect on protein unfolding (14). Various denaturants interact with protein in a variety of distinct mechanisms. As a result, the cooperativity of these interactions are critical to understanding the complete effect of the denaturant on each protein of interest. This is especially true at lower concentrations of denaturant, where the degree of cooperativity yields a large effect on the rate of denaturation of the protein. This measure of cooperativity, defined herein as the $\mu$-coefficient, for the influence of urea on protein denaturation was calculated to be $2.55 \pm 0.17$ for HEWL and $5.65 \pm 0.62$ for $\mathrm{HK}$. These results suggest that urea has a more pronounced effect on the rate of HEWL unfolding compared with HK.

The rate of unfolding for a given protein can be influenced by the choice

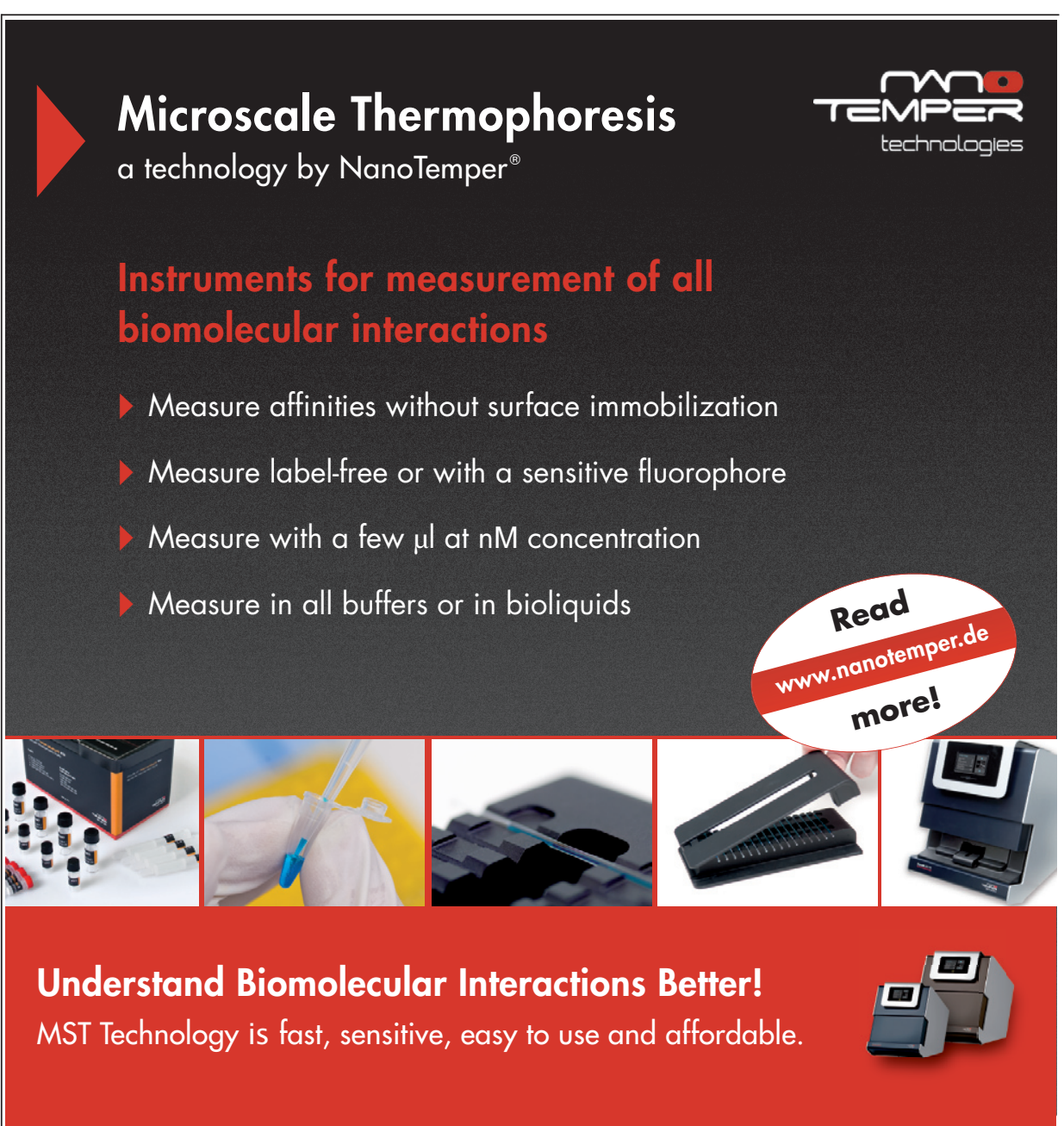

of chemical denaturant as well as by environmental factors such as temperature and $\mathrm{pH}$. One important advantage of our thermocycler-based method is the ability to utilize the thermal block for very precise control over sample temperature as well as the capacity to look for interacting effects of chemical denaturant and temperature. Many gradient-enabled thermocyclers can incubate and monitor samples at a wide range of temperatures in a single assay. For example, for a given experiment, the BioRad MyIQ2 thermal block can accommodate a temperature range of $25^{\circ} \mathrm{C}$ within the limits of $4^{\circ} \mathrm{C}$ and $99^{\circ} \mathrm{C}$. Using this ability to monitor fluorescent changes at multiple temperatures, we assessed the influence of temperature on the $\mathrm{K}_{\text {nd }}$ of urea for HEWL and $\mathrm{HK}$ in $10^{\circ} \mathrm{C}$ increments in separate trials over the range of $5^{\circ} \mathrm{C}$ to $35^{\circ} \mathrm{C}$ (Figure $2 \mathrm{C}$ and $3 \mathrm{C}$ ). No significant influence of temperature on $\mathrm{K}_{\text {nd }}$ was seen between $5^{\circ} \mathrm{C}$ and $35^{\circ} \mathrm{C}(P<0.05)$ for HEWL and no significant influence of temperature on $\mathrm{K}_{\text {nd }}$ was seen between $5^{\circ} \mathrm{C}$ and $15^{\circ} \mathrm{C}$ $(P<0.05)$ for $\mathrm{HK}$. These regions may represent temperature ranges in which 
$\mathrm{K}_{\mathrm{nd}}$ is independent of an increase in temperature. Indeed, previous studies have shown that lysozyme is thermostable up to $\sim 40^{\circ} \mathrm{C}$ and is $50 \%$ denatured at $\sim 50^{\circ} \mathrm{C}(15)$. However, at higher temperatures, $25^{\circ} \mathrm{C}$ and $35^{\circ} \mathrm{C}$, the $\mathrm{K}_{\text {nd }}$ for urea of HK significantly decreased and suggests a combined influence of denaturant (urea) and temperature on protein unfolding. The effect was pronounced, with $\mathrm{K}_{\mathrm{nd}}$ dropping from $\sim 5 \mathrm{M}$ at the lower temperatures to $\sim 1 \mathrm{M}$ at $35^{\circ} \mathrm{C}$, demonstrating that urea is a much more effective denaturant at higher temperatures. Perhaps this is because the native conformation of the protein at $35^{\circ} \mathrm{C}$ would be expected to be freer because of the higher temperature on protein and/or differential effects of high temperature on the hydrophobic versus hydrophilic bonds that underlie protein folding and conformation (16).

When comparing the experimentally derived $\mathrm{K}_{\mathrm{nd}}(2.69 \pm 0.06 \mathrm{M}$ urea $)$ to literature values of $\mathrm{C}_{1 / 2}(5.69 \pm 0.14$ $\mathrm{M}$ urea; where $\mathrm{C}_{1 / 2}$ is the urea concentration at which protein is $50 \%$ unfolded) for HEWL in urea, the experimentally derived $\mathrm{K}_{\text {nd }}$ is significantly different than the experimentally determined $\mathrm{C}_{1 / 2}$ value (17). Although these results are measurements of different unfolding characteristics of HEWL (50\% rate versus $50 \%$ amount unfolded protein), these results together may suggest that the rate of protein unfolding reaches the fastest possible rate of HEWL unfolding before the protein is completely unfolded. Interestingly, previous studies suggest that HEWL retains its overall structural conformation at urea concentrations below $2 \mathrm{M}$ urea (17). However, these studies determined relative protein unfolding by tryptophan fluorescence at $360 \mathrm{~nm}$ and the initial periods of unfolding may be below the limit of detection. In the case of native protein denaturation, hydrophobic residues that are exposed can interact and be detected with the SYPRO Orange dye. This may allow for a lower limit of detection, where the initial stages of protein unfolding can be detected, providing a better representation of the unfolding process. Native protein denaturation allows researchers to monitor the rate of unfolding in real-time during the entire progression, providing a greater resolution of the unfolding process.

Here we have presented a new technique to quantify the kinetics of protein denaturation. We obtained a half-maximal rate of native protein denaturation $\left(\mathrm{K}_{\mathrm{nd}}\right)$, maximum rate of denaturation $\left(D^{n d x}\right)$, and extent of denaturant cooperativity
( $\mu$-coefficient) under several experimental conditions. Our method is very simple, requires only small sample volumes, and provides large amounts of reproducible real-time data in a relatively short experimental protocol. Finally, this method will allow the researcher to quickly and efficiently determine the susceptibility of protein structure to many different denaturants while also allowing the variable of temperature change to be used as a means to further manipulate denaturation. Stability data could also be collected as a function of competing stabilizing agents. However, this study may be limited by the rate of SYPRO incorporation relative to extremely high rates of protein unfolding. Additionally, proteins must contain sufficient hydrophobic regions to interact with the SYPRO Orange dye; a property that will influence the sensitivity of the technique for each particular protein of interest. This method provides an opportunity for researchers to explore protein stability with readily available equipment, and allows current studies that evaluate protein stability to explore changes in degradation kinetics.

\section{Acknowledgments}

Thanks to J.M. Storey for an editorial review of the manuscript. This work was supported by a Discovery grant from the Natural Sciences and Engineering Research Council (NSERC) of Canada. K.B.S. holds the Canada Research Chair in Molecular Physiology, K.K.B. held an NSERC postgraduate fellowship.

\section{Competing interests}

The authors declare no competing interests.

\section{References}

1. Hendry, P. 2006. Extremophiles: There's more to life. Environ. Chem. 3:75-76.

2. Padan, E., D. Zilberstein, and S. Schuldiner. 1981. pH-Homeostasis in bacteria. Biochim. Biophys. Acta 650:151-166.

3. Baker-Austin, C. and M. Dopson. 2007. Life in acid: $\mathrm{pH}$ homeostasis in acidophiles. Trends Microbiol. 15:165-171.

4. Storey, K.B. 1997. Metabolic regulation in mammalian hibernation: Enzyme and protein adaptations. Comp. Biochem. Physiol. A Physiol. 118:1115-1124.

5. Ramnanan, C.J. and K.B. Storey. 2006. Glucose-6-phosphate dehydrogenase regulation during hypometabolism. Biochem. Biophys. Res. Commun. 339:7-16.

6. Ramnanan, C.J., A.G. Groom, and K.B. Storey. 2007. Akt and its downstream targets play key roles in mediating dormancy in land snails. Comp. Biochem. Physiol. B 148:245-255.
7. Dieni, C.A. and K.B. Storey. 2009. Creatine kinase regulation by reversible phosphorylation in frog muscle. Comp. Biochem. Physiol. B 152:405-412.

8. Niesen, F.H., H. Berglund, and M. Vedadi. 2007. The use of differential scanning fluorimetry to detect ligand interactions that promote protein stability. Nat. Protoc. 2:2212-2221.

9. Brooks, S.P.J. 1994. A program for analyzing enzyme rate data obtained from a microplate reader. BioTechniques 17:1154-1161.

10. Griko, Y.V., E. Freire, G. Privalov, H.V. Dael, and P.L. Privalov. 1995. The unfolding thermodynamics of c-type lysozymes: a calorimetric study of the heat denaturation of equine lysozyme. J. Mol. Biol. 252:447459.

11. Kumar, D.P., A. Tiwari, and R. Bhat. 2004. Effect of $\mathrm{pH}$ on the stability and structure of yeast hexokinase A: acidic amino acid residues in the cleft region are critical for the opening and the closing of the structure. J. Biol. Chem. 279:32093-32099.

12. Hofmeyr, J.S. and A. Cornish-Bowden. 1997. The reversible Hill equation: how to incorporate cooperative enzymes into metabolic models. Comput. Appl. Biosci. 13:377-385.

13. Laurents, D.V. and R.L. Baldwin. 1997. Characterization of the unfolding pathway of hen egg white lysozyme. Biochemistry 36:1496-1504.

14. Kim, K.S. and C. Woodward. 1993. Protein internal flexibility and global stability: Effect of urea on hydrogen exchange rates of bovine pancreatic trypsin inhibitor. Biochemistry 32:9609-13.

15. Bonincontro, A., S. Cinelli, T. Comaschi, and G. Onori. 2004. Influence of urea on thermal denaturation of lysozyme investigated by optical dielectric spectroscopies. Phys. Chem. Chem. Phys. 6:1039-1042.

16. Hochachka, P.W. and G.N. Somero. 1984. Biochemical Adaptation. Princeton University Press, Princeton, NJ.

17. Ibarra-Molero, B. and J.M. Sanchez-Ruiz. 1997. Are There Equilibrium Intermediate States in the Urea-Induced Unfolding of Hen Egg-White Lysozyme? Biochemistry 36:9616-9624.

Received 1 May 2012; accepted 13 September 2012.

Address correspondence to Kenneth B. Storey, Institute of Biochemistry \& Department of Biology, Carleton University, 1125 Colonel By Drive, Ottawa, Ontario, Canada. Email: kenneth_storey@carleton.ca

To purchase reprints of this article, contact: biotechniques@fosterprinting.com 